\title{
Nanometer-scale studies of Al-Ga interdiffusion and As precipitate coarsening in nonstoichiometric AIAs/GaAs superlattices
}

\author{
B. Lita, Smita Ghaisas, and R. S. Goldman ${ }^{\text {a) }}$ \\ Department of Materials Science and Engineering, University of Michigan, Ann Arbor, Michigan 48109 \\ M. R. Melloch \\ School of Electrical and Computer Engineering, Purdue University, West Lafayette, Indiana 47907
}

(Received 31 August 1999; accepted for publication 2 November 1999)

\begin{abstract}
We have investigated the effects of post-growth annealing on $\mathrm{Al}-\mathrm{Ga}$ interdiffusion and As precipitate coarsening in $\mathrm{AlAs} / \mathrm{GaAs}$ superlattices grown by molecular-beam epitaxy at low temperatures. High-resolution x-ray diffraction spectra show a significant decrease in the number and intensity of satellite peaks for the ex situ annealed compared with the as-grown superlattices, a feature which is often attributed to a reduction in interface abruptness. However, our cross-sectional scanning tunneling microscopy images show significant variation in the apparent superlattice period of the ex situ annealed compared with the as-grown superlattices. For the as-grown superlattices, preferential As precipitation on the GaAs side of AlAs/GaAs interfaces is evident. In the ex situ annealed superlattices, a preference for As precipitates at the GaAs on AlAs interface is apparent, although the As precipitates are no longer restricted to the interface region. Thus, the apparent change in superlattice period is likely due to variations in As precipitate density, which may be influenced by AlAs-GaAs alloying at the AlAs/GaAs interfaces. (C) 1999 American Institute of Physics. [S0003-6951(99)00152-7]
\end{abstract}

Compound semiconductor films grown by molecularbeam epitaxy at low temperatures $\left(200-450{ }^{\circ} \mathrm{C}\right)$ are generally nonstoichiometric. ${ }^{1,2}$ Excess arsenic (As) is incorporated as As antisite defects $\left(\mathrm{As}_{\mathrm{Ga}}\right)$ and As interstitials, often thought to be accompanied by a supersaturation of gallium vacancies. ${ }^{3,4}$ Upon annealing above the growth temperature, the excess As segregates, forming a mixture of GaAs and semimetallic As precipitates which deplete the surrounding regions of free carriers. With additional annealing, the As precipitates coarsen, increasing the size of the depleted regions. The amount of excess As in the epilayers is controlled by the growth temperature, and the average size and density of As precipitates are controlled by the post-growth annealing temperature. ${ }^{1}$ The ability to control the density and distribution of excess As in compound semiconductors has opened up new possibilities for tuning the resistivity over a wide range from $0.3 \Omega$-cm for ultrapure intrinsic GaAs to $10^{11} \Omega$-cm for low temperature grown AlGaAs. ${ }^{5}$ Hence, it is not surprising that many new device applications have emerged, including photorefractive semiconductors for holographic applications, ${ }^{6}$ nonalloyed ohmic contacts, ${ }^{7}$ ultrafast photodetectors, ${ }^{8,9}$ and patterned self-organized devices. ${ }^{10}$ The nanometer-scale mechanisms of $\mathrm{Al}-\mathrm{Ga}$ interdiffusion and As precipitate coarsening occurring during the postgrowth annealing steps critical for devices are not well understood. Therefore, we have investigated the effects of postgrowth annealing on the structure of $\mathrm{AlAs} / \mathrm{GaAs}$ superlattices grown at low temperature, using ultrahigh vacuum cross-sectional scanning tunneling microscopy (XSTM) and high-resolution X-ray diffraction. In accordance with earlier work, ${ }^{11}$ we find a significant decrease in the intensity and number of satellite peaks for the ex situ annealed superlattices, in comparison with the as-grown super-

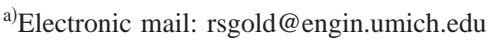

lattices. This type of trend has been observed in many superlattice systems and is generally attributed to a reduction in interface abruptness due to interface intermixing. Here, our XSTM studies reveal an additional significant effect: a difference in the apparent superlattice periods of the as-grown and $e x$ situ annealed superlattices. For the as-grown superlattices, arsenic precipitates tend to be lined up along the GaAs side of each AlAs/GaAs interface. After ex situ annealing, this preferential As precipitation is less apparent. It is likely that AlAs-GaAs alloying at the AlAs/GaAs interfaces has diminished the preferential As precipitation, and that the difference in apparent superlattice periods may be explained by the resulting variations in As precipitate distributions.

For these investigations, Si-doped $\left(N_{d} \sim 1\right.$ $\times 10^{18} \mathrm{~cm}^{-3}$ ) heterostructures consisting of 75-period superlattices of alternating $10 \mathrm{~nm} n$-GaAs quantum wells and $3.5 \mathrm{~nm} n$-AlAs barriers were grown by solid source molecular-beam epitaxy at $325{ }^{\circ} \mathrm{C}$ on a (001)-oriented Sidoped $\left(N_{d} \sim 5 \times 10^{18} \mathrm{~cm}^{-3}\right)$ GaAs substrate. The superlattices were sandwiched between $50 \mathrm{~nm} n$-AlGaAs layers grown at $450{ }^{\circ} \mathrm{C}$. Since the superlattices were effectively in situ annealed for approximately $6 \mathrm{~min}$ at $450{ }^{\circ} \mathrm{C}$, it is likely that most of the excess As segregated to form precipitates. ${ }^{12}$ After growth, some of the samples were rapid thermal annealed in nitrogen gas at $900{ }^{\circ} \mathrm{C}$ for $30 \mathrm{~s}$. We will refer to the AlAs/GaAs superlattices before and after post-growth annealing cycles as "as-grown" and "ex situ annealed," respectively.

High-resolution $\mathrm{x}$-ray rocking curves (XRC) were measured by double-axis x-ray diffraction using $\mathrm{Cu} K_{\alpha}$ radiation monochromated by a four-reflection Bartels monochromator, which employs $\mathrm{Ge}(220)$ reflections in the $(+,-,-,+)$ configuration. The rocking curves were collected near the $\mathrm{GaAs}(002)$ reflection. For XSTM, the samples were cleaved to expose a (110) surface, in an ultrahigh vacuum (UHV) 


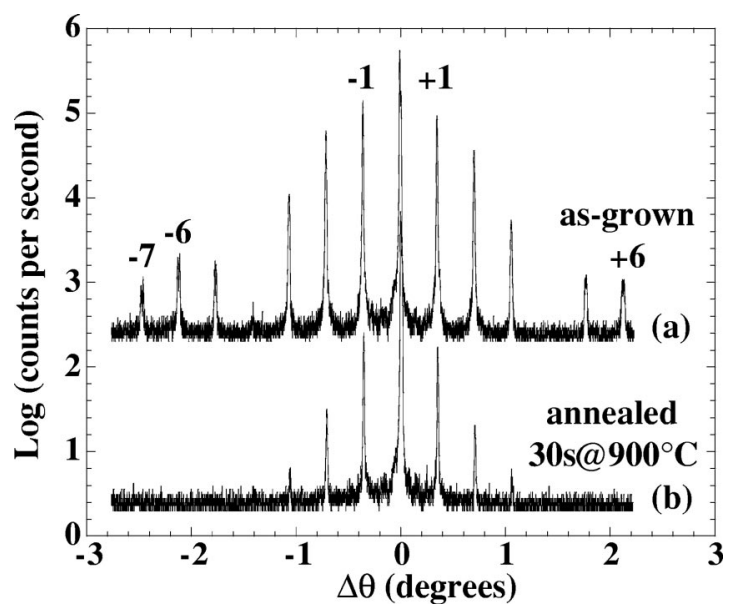

FIG. 1. High-resolution X-ray rocking curves, collected near the GaAs(002) reflection of (a) as-grown and (b) ex situ annealed superlattices.

chamber with base pressure $\leqslant 5 \times 10^{-11}$ Torr. ${ }^{13}$ All images were obtained with a constant tunnel current of $0.2 \mathrm{nA}$ unless specified otherwise, and sample bias voltages ranging from -1.55 to $-3 \mathrm{~V}$.

Figures 1(a) and 1(b) show (002) high-resolution x-ray rocking curves of (a) as-grown and (b) ex situ annealed superlattices. The presence of satellite peaks up to at least the sixth order in Fig. 1(a) suggests that the as-grown superlattices possess a well-defined periodic structure. The average spacing between the satellite peaks indicates an average superlattice period of $13.0 \pm 0.1 \mathrm{~nm}$ in both cases. The absence of satellite peaks past the third order in (b) suggests that the post-growth annealing process has led to a less well-defined periodic structure. In earlier work, similar results were attributed to reduced interface abruptness after ex situ annealing. ${ }^{11}$ We will show that the situation is actually further complicated by variations in the distributions of As precipitates.

Differences in the apparent superlattice periods of the as-grown and ex situ annealed superlattices are evident in the large-scale XSTM topographic images shown in Figs. 2(a) and 2(b), respectively. In both images, alternating bright and dark regions, corresponding to GaAs and AlAs layers, are apparent. The brightest spots are attributed to As precipitates, similar to earlier reports. ${ }^{14,15}$ In the as-grown superlattices shown in Fig. 2(a), the superlattice period appears constant with an average value of $13.2 \pm 0.1 \mathrm{~nm}$, similar to the $13 \mathrm{~nm}$ period determined from XRC. In Fig. 2(b), several periods of the ex situ annealed superlattice are presented. In this case, the GaAs layers appear inhomogeneous, due to AlAs-GaAs alloying and/or to the presence of a nonuniform density of As precipitates. Furthermore, the superlattice period is nonconstant, and may appear larger or smaller than the average value determined by XRC. As will be discussed in more detail below, this change in the apparent superlattice period may be due to differences in the distribution of As precipitates, rather than a significant variation in the AlAs/ GaAs superlattice period.

In the as-grown superlattices, the As precipitates are typically spherical, with diameters ranging from 1 to $5 \mathrm{~nm}$, as shown in Fig. 2(a). A small fraction of the precipitates appear oval shaped, presumably due to the agglomeration or apparent agglomeration of two or more precipitates. Assuming that the cleave has bisected precipitates with an average diameter of $2.5 \mathrm{~nm}$, we estimate a precipitate density of 5.2 $\times 10^{16} \mathrm{~cm}^{-3}$. In earlier cross-sectional transmission electron microscopy observations of nonstoichiometric GaAs annealed at $600{ }^{\circ} \mathrm{C}$ for $1 \mathrm{~h},{ }^{16}$ the precipitate diameters ranged from 2.5 to $8 \mathrm{~nm}$, with an average precipitate density of $4.6 \times 10^{15} \mathrm{~cm}^{-3}$. It is expected that annealing nonstoichiometric GaAs at a lower temperature and for a shorter time would lead to a much higher density of smaller arsenic precipitates, ${ }^{1}$ which is precisely what we have observed in the present study.

Most of the precipitates in the as-grown superlattices are lined up along the GaAs side of each AlAs/GaAs interface. For example, in Fig. 2(a), 85\% of the precipitates are situated along the GaAs side of the interface, while only $11 \%$ and $4 \%$ are located within the GaAs and AlAs layers, respectively. The preference for arsenic to precipitate in GaAs has been explained by the lower $\mathrm{Ga}-\mathrm{As}$ bond energy compared with that of $\mathrm{Al}-\mathrm{As},{ }^{17}$ which results in a lower interfacial energy

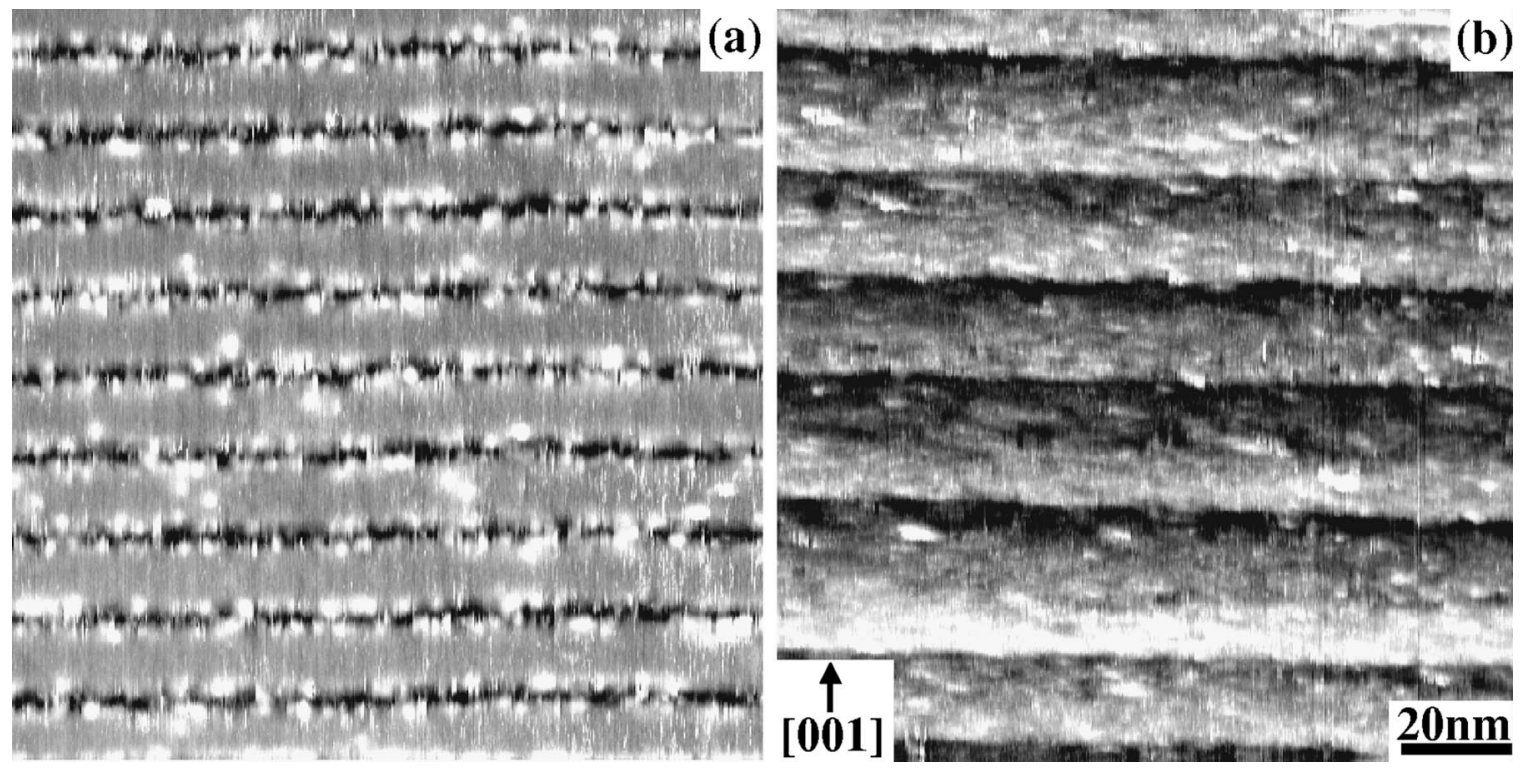

FIG. 2. Large-scale topographic images of (a) as-grown and (b) ex situ annealed superlattices, obtained with (a) $0.2 \mathrm{nA}$ and (b) $0.5 \mathrm{nA}$ tunneling currents and (a) $-1.8 \mathrm{~V}$ and (b) $-3 \mathrm{~V}$ sample bias voltages. The gray-scale ranges displayed are (a) $4.7 \AA$ and (b) $5 \AA$. 


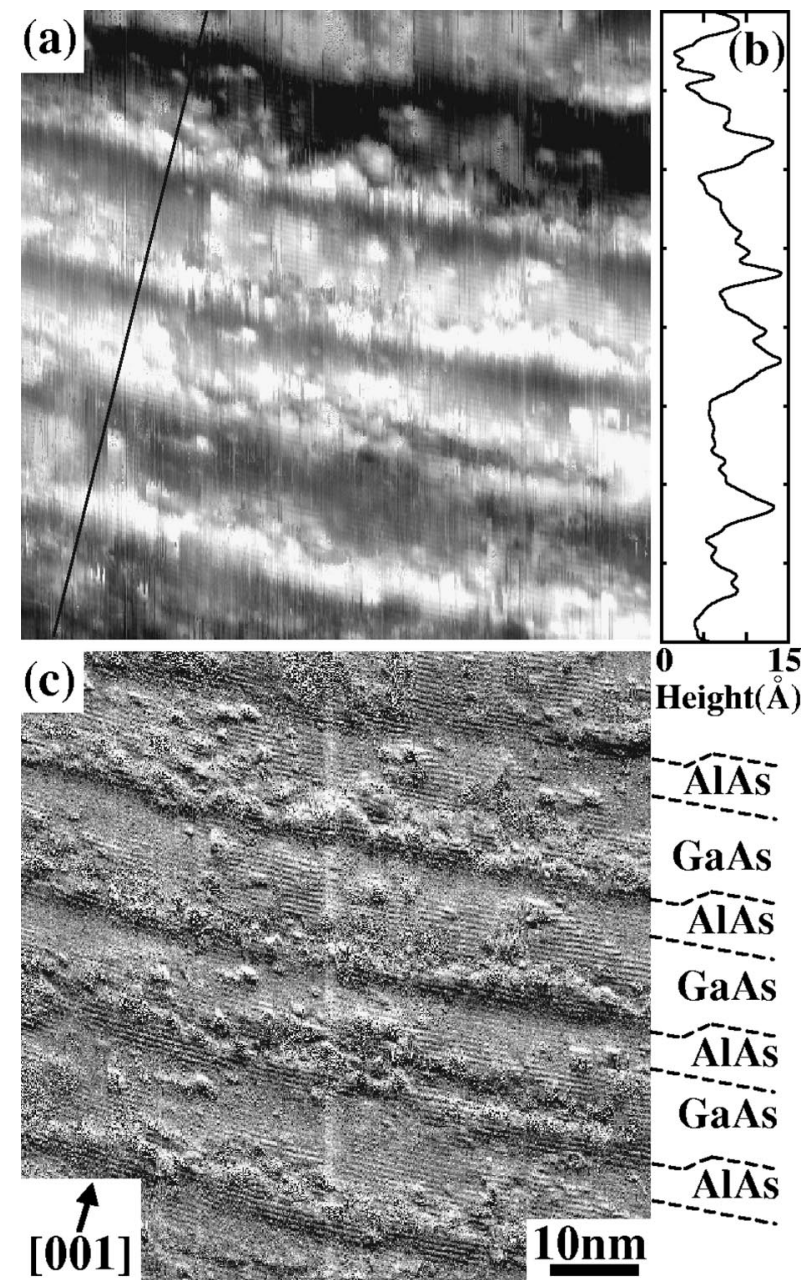

FIG. 3. High-resolution (a) topographic image and (c) corresponding current image of ex situ annealed superlattices, obtained simultaneously at a $-1.55 \mathrm{~V}$ sample bias voltage. The gray-scale ranges displayed are (a) $4.2 \AA$ and (c) $0.14 \mathrm{nA}$. A cut of tip height along the line indicated in (a), with the $y$ coordinate compressed by $3.1 \%$, is displayed in (b).

of the As precipitate in GaAs compared with that of the As precipitate in AlAs. ${ }^{18}$ The smaller interfacial energy of the precipitates in GaAs is expected to lead to a lower nucleation barrier and to more significant coarsening of the As precipitates in GaAs than in AlAs. ${ }^{19}$ The preferential As precipitation along the AlAs/GaAs interface, which has not been explained previously, may be due to the presence of lattice distortions at the interface. Since the AlAs lattice constant is larger than that of GaAs, the AlAs and GaAs lattices are distorted in compression and tension, respectively. The As precipitates would most easily fit into a lattice which is in tension, and would prefer to be located where the tensile distortion is highest, which occurs in the GaAs, close to the $\mathrm{AlAs} / \mathrm{GaAs}$ interface. A similar alignment of As precipitates was observed on the Be-doped GaAs side of Be-doped GaAs/GaAs interface. ${ }^{20}$

In the ex situ annealed superlattices, the As precipitates which were initially lined up along AlAs/GaAs interfaces have coarsened more significantly than those in other locations. In addition, a preference for As precipitates at the GaAs on AlAs interface is observed, as shown in the topography and current images in Figs. 3(a) and 3(c). In Fig. 3(b), the cut of tip height along the line in Fig. 3(a), narrow depression (valleys) and protrusions (peaks), corresponding to
AlAs and As precipitates, are apparent. The As precipitates also appear as protrusions in the current image displayed in Fig. 3(c). Furthermore, the current image in Fig. 3(c) suggests a higher density of As precipitates near the GaAs on AlAs interfaces. This is likely to be related to the differences in abruptness between GaAs on AlAs and AlAs on GaAs interfaces, which have been reported in the literature. ${ }^{21} \mathrm{Fi}$ nally, the appearance of the ex situ annealed superlattices is often altered by the presence of bright elongated features which presumably consist of agglomerations of As precipitates. An example of such an elongated feature is apparent towards the bottom of Fig. 2(b). Features of this type have been observed in many regions of the ex situ annealed superlattices, with tip heights ranging from 1.9 to $9.6 \AA$ above the nominal GaAs height. Taken together, these observations suggest that the apparent change in superlattice period in the ex situ annealed superlattice is due to a variation in the As precipitate distribution, which is presumably strongly influenced by AlAs-GaAs alloying at the interfaces.

The authors would like to thank K. R. Nikolaev for collecting the $\mathrm{x}$-ray diffraction data shown in Fig. 1. This work was supported in part by the Office of the Provost, the Office of the Vice President for Research, and the Rackham Graduate School, at the University of Michigan, the donors of the Petroleum Research Fund, administered by the American Chemical Society, the Dow Corning Foundation, and the Air Force Office of Scientific Research.

${ }^{1}$ M. R. Melloch, D. D. Nolte, J. M. Woodall, J. C. P. Chang, D. B. Janes, and E. S. Harmon, Crit. Rev. Solid State Mater. Sci. 21, 189 (1996).

${ }^{2}$ D. C. Look, Thin Solid Films 231, 61 (1993).

${ }^{3}$ D. E. Bliss, W. Walukiewicz, J. W. Ager III, E. E. Haller, K. T. Chan, and S. Tanigawa, J. Appl. Phys. 71, 1699 (1992).

${ }^{4}$ C. Kisielowski, A. R. Calawa, and Z. Liliental-Weber, J. Appl. Phys. 80, 156 (1996).

${ }^{5}$ G. L. Witt, Mater. Sci. Eng., B 22, 9 (1993).

${ }^{6}$ D. D. Nolte and M. R. Melloch, Mater. Res. Bull. 19, 44 (1994).

${ }^{7}$ P. D. Kirchner, T. N. Jackson, G. D. Pettit, and J. M. Woodall, Appl. Phys. Lett. 47, 26 (1985).

${ }^{8}$ F. W. Smith, H. Q. Le, V. Diadiuk, M. A. Hollis, A. R. Calawa, S. Gupta, M. Frankel, D. R. Dykaar, G. A. Mourou, and T. Y. Hsiang, Appl. Phys. Lett. 54, 890 (1989).

${ }^{9}$ E. S. Harmon, M. R. Melloch, J. M. Woodall, D. D. Nolte, N. Otsuka, and C. L. Chang, Appl. Phys. Lett. 63, 2248 (1993).

${ }^{10}$ R. A. Kiehl, M. Yamaguchi, O. Ueda, N. Horiguchi, and N. Yokoyama, Appl. Phys. Lett. 68, 478 (1996).

${ }^{11}$ J. C. P. Chang, J. M. Woodall, M. R. Melloch, I. Lahiri, D. D. Nolte, N. Y. Li, and C. W. Tu, Appl. Phys. Lett. 67, 3491 (1995).

${ }^{12}$ M. R. Melloch, I. Lahiri, D. D. Nolte, J. C. P. Chang, E. S. Harmon, J. M. Woodall, N. Y. Li, and C. W. Tu, J. Vac. Sci. Technol. B 14, 2271 (1996).

${ }^{13}$ B. Lita, R. S. Goldman, J. D. Phillips, and P. K. Bhattacharya, Appl. Phys. Lett. 74, 2824 (1999).

${ }^{14}$ R. M. Feenstra, J. M. Woodall, and G. D. Pettit, Phys. Rev. Lett. 71, 1176 (1993).

${ }^{15}$ R. M. Feenstra, A. Vaterlaus, J. M. Woodall, and G. D. Pettit, Appl. Phys. Lett. 63, 2528 (1993).

${ }^{16}$ K. Mahalingam, N. Otsuka, M. R. Melloch, J. M. Woodall, and A. C. Warren, J. Vac. Sci. Technol. B 9, 2328 (1991).

${ }^{17}$ O. Kubaschewski, C. B. Alcock, and P. J. Spencer, Materials Thermochemistry (Pergamon Press, Oxford, 1993).

${ }^{18}$ K. Mahalingam, N. Otsuka, M. R. Melloch, and J. M. Woodall, Appl. Phys. Lett. 60, 3253 (1992)

${ }^{19}$ P. W. Voorhees, J. Stat. Phys. 38, 232 (1985).

${ }^{20}$ Z. A. Su, J. H. Huang, L. Z. Hsieh, and W.-I. Lee, Appl. Phys. Lett. 72, 1984 (1998)

${ }^{21}$ M. Tanaka, T. Noda, and H. Sakaki, Mater. Sci. Eng. B 14, 304 (1992), and references therein. 